\title{
Abnormal Localization and Accumulation of FLT3-ITD, a Mutant Receptor Tyrosine Kinase Involved in Leukemogenesis
}

\author{
Sina Koch ${ }^{\mathrm{a}}$ Angela Jacobi ${ }^{\mathrm{b}}$ Martin Ryser ${ }^{\mathrm{b}} \quad$ Gerhard Ehninger $^{\mathrm{a}}$ \\ Christian Thiede ${ }^{a}$ \\ ${ }^{a}$ Medizinische Klinik und Poliklinik I and ${ }^{b}$ Klinik und Poliklinik für Pädiatrie, Universitätsklinikum Carl Gustav Carus \\ der Technischen Universität Dresden, Dresden, Deutschland
}

\section{Key Words}

Acute myeloid leukemia • Receptor tyrosine kinase •

FLT3-ITD · Subcellular localization

\begin{abstract}
Aberrant subcellular localization of mutant transmembrane receptors is increasingly acknowledged as a possible mechanism for an altered signaling quality leading to transformation. There is evidence that mutated receptor tyrosine kinases of subclass III, for example the platelet-derived growth factor receptor (PDGFR) and KIT-protein, are aberrantly localized in human cancers. In order to further analyze this phenomenon, we investigated the localization of FLT3, a subclass III receptor tyrosine kinase frequently mutated in leukemia. By immunofluorescence staining and confocal laser scanning microscopy we found that in retrovirally transduced COS7 cells, wild type FLT3 receptor protein is localized primarily at the cell surface. In contrast, a mutant FLT3 receptor protein with an internal tandem duplication (ITD) accumulates in a perinuclear region and is not detectable at the plasma membrane. Surprisingly, and in contrast to previously published data, intracellular FLT3-ITD accumulation could neither be detected in the endoplasmic reticulum (ER) nor in the Golgi apparatus. Furthermore, transient overexpression per se leads to accumulation of wild type FLT3 receptor pro-
\end{abstract}

tein in the ER in addition to surface localization, probably due to inefficient intracellular transport by the overloaded sorting machinery of the secretory pathway. Based on our data and the immature glycosylation pattern of FLT3-ITD, we speculate that the mutant protein resides most probably in an unidentified compartment of the secretory pathway between the ER and the Golgi apparatus.

Copyright $\odot 2008$ S. Karger AG, Base

\begin{tabular}{ll}
\hline Abbreviations used in this paper \\
\hline AML & acute myeloid leukemia \\
CSF-1 & colony stimulating factor 1 \\
ECFP & endopced cyan fluorescent protein \\
ER & FLT3 ligand \\
FL & FMS-like Tyrosine kinase 3 \\
FLT3 & friend murine leukemia virus integration site 2 \\
FMS & internal tandem duplication \\
ITD & juxtamembrane domain \\
JMD & platelet-derived growth factor receptor \\
PDGFR & receptor tyrosine kinase \\
RTK & stem cell factor \\
SCF & tyrosine kinase domain \\
TKD & wheat germ agglutinin \\
WGA & wild type \\
wt &
\end{tabular}

\section{KARGER}

Fax +4161306 1234

E-Mail karger@karger.ch

www.karger.com
C 2008 S. Karger AG, Basel

$1422-6405 / 08 / 1882-0225 \$ 24.50 / 0$

Accessible online at:

www.karger.com/cto
Dr. Christian Thiede

Medizinische Klinik und Poliklinik I

Universitätsklinikum Carl Gustav Carus der Technischen Universität Dresden

Fetscherstrasse 74, DE-01307 Dresden (Germany)

Tel. +49351 458 4680, Fax +49351 458 5362, E-Mail thiede@mk1.med.tu-dresden.de 


\section{Introduction}

Cytokine signaling via transmembrane receptors is a critical regulatory element in tissue formation, differentiation and the regulation of cell proliferation. Among these transmembrane receptors, several different groups of cognate receptors (for example, receptor and nonreceptor tyrosine kinases, $G$ protein-coupled receptors) have been described to be relevant for many human diseases, including cancer. One large group of these receptors comprises the receptor tyrosine kinases (RTKs). Based on bioinformatic analysis of the human genome, 58 RTKs have been identified, which are assorted into 20 subfamilies, based on structural motifs [Robinson et al., 2000].

The RTKs of subclass III are widely expressed in normal human tissues, where they are involved in a variety of functions, such as regulation of cell growth, proliferation, migration, differentiation and apoptosis [BlumeJensen and Hunter, 2001; Grassot et al., 2003]. The subclass III family of RTKs comprises the colony stimulating factor 1 (CSF-1) receptor friend murine leukemia virus integration site 2 (FMS), the stem cell factor (SCF) receptor KIT, the platelet-derived growth factor receptors (PDGFR) $\alpha$ and $\beta$ and the FMS-like tyrosine kinase 3 (FLT3), which share common structural features, that is, five extracellular immunoglobulin-like domains, one transmembrane domain, a juxtamembrane domain (JMD) and an intracellular tyrosine kinase domain (TKD), which is interrupted by a kinase insert domain [van der Geer et al., 1994; Grassot et al., 2003].

In the inactive receptors, the activation loop within the TKD stably assumes the closed conformation by folding into the cleft between a bilobal kinase fold [Huse and Kuriyan, 2002], and the JMD maintains a bound conformation supporting the autoinhibitory state [Mol et al., 2004; Walter et al., 2007], thereby blocking access for ATP and peptide substrates. Ligand binding promotes dimerization of two receptor monomers and subsequently tyrosine residues in the JMD are transphosphorylated [Rosnet and Birnbaum, 1993; Heldin, 1995; Turner et al., 1996]. The charged bulky phosphate moieties on tyrosine residues in the JMD prevent folding of the JMD and consequently the autoinhibited state [Griffith et al., 2004]. As a result, phosphorylation of typically 1-3 tyrosine residues in the activation loop provides access for protein substrates [Huse and Kuriyan, 2002].

Constitutively activating mutations of RTK of subclass III have been found in different human cancers and lead to permanent induction of downstream signaling. For instance, activating mutations in the FMS gene have been reported in human myelodysplastic syndromes and acute myeloid leukemia (AML) [Tobal et al., 1990] as well as in hepatocellular carcinoma [Cui et al., 2001; Yang et al., 2004] and in human gynecological cancers, such as of the uterus, ovaries and cervix [Kirma et al., 2007]. Mutations of the KIT receptor and, although less frequent, PDGFR $\alpha$ are considered to be crucial events in the pathogenesis of gastrointestinal stromal tumors [Rubin et al., 2001; Antonescu et al., 2003; Heinrich et al., 2003]. Furthermore, the most frequent mutation in systemic mastocytosis is a constitutively activating mutation in the KIT gene [Nagata et al., 1995; Longley et al., 1996], and an activating transforming mutation in the PDGFR $\alpha$ sequence was identified in primary human glioblastoma tissue [Kumabe et al., 1992; Clarke and Dirks, 2003].

Mutated FLT3 is one of the most frequent somatic alterations in AML and mutations occur in approximately $30 \%$ of patients. FLT3 mutations consist of two major types: internal tandem duplications (ITD) mapping to the JMD (approx. 20\% of AML patients) and point mutations which most frequently involve D835 of the TKD (8-12\%) [Yamamoto et al., 2001]. Although both types of mutation result in constitutive ligand-independent activation of the FLT3 receptor, the mechanisms of activation, the downstream signaling pathways [Choudhary et al., 2005] and the resulting diseases [Grundler et al., 2005] appear to be different. TKD mutations are thought to destabilize the activation loop, bringing it into an active open conformation [Griffith et al., 2004], and subsequent signaling pathways are similar to FLT3wt [Choudhary et al., 2005]. The precise mechanism of receptor activation by ITD mutations is unknown, but it is speculated that ITD mutations disrupt the intrinsic negative regulatory effects of the JMD on its own TKDs, whereby ITD mutations change the conformation of the monomeric receptor, exposing the phosphoryl acceptor sites in the TKDs [Kiyoi et al., 2002].

Besides these intramolecular mechanisms for constitutive activation, aberrant localization of mutated RTKs has been reported [Tang et al., 1990; Kacinski et al., 1991; Miettinen and Lasota, 2001; Clarke and Dirks, 2003; Xiang et al., 2007], and has been suggested to be an important mechanism for their abnormal signaling. Recently, a study reported that FLT3-ITD has an impact on the maturation of the receptor as well as on its intracellular localization and discussed this as the potential mechanism for initiating different signaling cascades [Schmidt-Arras et al., 2005]. In this study, the authors showed that mutant FLT3ITD protein localizes to the endoplasmic reticulum (ER) and the Golgi apparatus, whereas the FLT3wt protein was localized mainly in the plasma membrane. 
In our study, we further investigated the localization of the mutant FLT3-ITD receptor in COS7 cells and additionally of the FLT3wt receptor for comparison. We could show the surface localization of FLT3wt receptor protein and confirm the aberrant predominantly intracellular accumulation of FLT3-ITD receptor protein. However, in contrast to Schmidt-Arras et al. [2005], our data show that FLT3-ITD receptor protein is neither localized in the ER nor in the Golgi apparatus, but in an additional, unidentified compartment.

\section{Materials and Methods}

\section{Growth Factors and Antibodies}

Recombinant human FLT3 ligand and mouse monoclonal anti-human FLT3 antibody (epitope in the extracellular part of FLT3) were purchased from R\&D Systems (Minneapolis, Minn., USA). Wheat germ agglutinin (WGA) conjugated to Alexa Fluor 488 and Alexa Fluor 488-conjugated anti-rabbit IgG were purchased from Molecular Probes (Eugene, Oreg., USA). Mouse monoclonal anti-phosphoFLT3 antibody was purchased from Cell Signaling Technology (Danvers, Mass., USA). Mouse monoclonal anti-actin antibody, sheep anti-mouse IgG-Cy3 conjugate, rabbit anti-calnexin antibody and $\mathrm{Cy} 3$-conjugated anti-mouse IgG antibody were purchased from Sigma Aldrich (St. Louis, Mo., USA). Rabbit polyclonal anti-giantin antibody was purchased from Covance (Princeton, N.J., USA). Horseradish peroxidase-conjugated anti-mouse secondary antibody was purchased from Dako Cytomation (Glostrup, Denmark). Cy5-conjugated anti-rabbit IgG antibody was purchased from Abcam (Cambridge, UK).

\section{Cell Line}

COS7 cells were purchased from the German Collection of Microorganisms and Cell Cultures (DSMZ, Braunschweig, Germany) and cultured in Dulbecco's modified Eagle medium (Gibco, Karlsruhe, Germany) supplemented with $10 \%$ (v/v) heat-inactivated FCS (Gibco) and 1\% (v/v) penicillin/streptomycin (PAA Laboratories, Pasching, Austria) in a humidified incubator at $37^{\circ} \mathrm{C}$ and $5 \% \mathrm{CO}_{2}$.

\section{DNA Constructs}

The genes for FLT3wt and FLT3-ITD were cloned from the human leukemic cell lines EOL-1 and MV4-11, respectively. Total RNA was isolated with the RNeasy Mini Kit from Qiagen (Hilden, Germany) according to the manufacturer's instructions. FLT3wt and FLT3-ITD cDNAs were obtained by reverse transcription with the Advantage ${ }^{\mathrm{TM}} \mathrm{RT}$-for-PCR kit from BD Biosciences (Palo Alto, Calif., USA) as recommended by the manufacturer. The correct sequences of the constructs were verified by direct sequencing in both directions.

\section{Retroviral Transduction of COS7 Cells}

FLT3 constructs were subcloned into a $\gamma$-retroviral MFG vector [Riviere et al., 1995] that contained an IRES-EGFP site to obtain MFG-FLT3wt-IRES-GFP and MFG-FLT3-ITD-IRES-GFP. VSVg-pseudotyped virus supernatant for both constructs was produced in $293 \mathrm{~T}$ cells and used to transduce FLYRD18 packaging cells [Cosset et al., 1995]. Producer clones were selected and vector virus (MFG-FLT3wt-IRES-GFP and MFG-FLT3-ITDIRES-GFP) was harvested from the supernatant. Concentrated virus was used to transduce COS7 cells.

\section{Transient Transfection of COS7 Cells}

FLT 3 constructs were subcloned into $\mathrm{pDONR}^{\mathrm{TM}}$ and pT-RExDEST Gateway ${ }^{\mathrm{TM}}$ vectors (Invitrogen, Paisley, UK) and transiently transfected into COS7 cells with Lipofectamine ${ }^{\mathrm{TM}} 2000$ (Invitrogen) according to the manufacturer's instructions.

\section{High-Resolution Denaturing Fragment Analysis}

The expression of either FLT3wt or FLT3-ITD in COS7 cells was verified by extracting DNA from cells and using gel electrophoresis of the fluorescently labeled PCR products generated from amplifying exons 14 and 15 of the FLT3 gene, in which the ITD mutation occurs. The PCR product of mutant FLT3-ITD is $30 \mathrm{bp}$ longer than that of FLT3wt due to the ITD insertion. Fluorescent PCR products were separated according to size by electrophoretic migration through a denaturing gel. Fluorescence intensity of the products was detected by laser excitation in an ABI 377 automatic sequencing machine (Applied Biosystems, Foster City, Calif., USA) and signals were integrated by Genescan software 3.1 (Applied Biosystems).

\section{Protein Extraction and Western Blot Analysis}

Cells were lysed in lysis buffer $(10 \mathrm{~mm}$ Tris/ $\mathrm{HCl} \mathrm{pH} 7.5,130$ mM sodium chloride, 5 mM EDTA pH 8.0, 1\% Triton X-100, 50 $\mathrm{mm}$ sodium fluoride, $10 \mathrm{~mm}$ sodium pyrophosphate, $20 \mathrm{~mm}$ disodium hydrogen phosphate, $20 \mathrm{~mm}$ sodium dihydrogen phosphate, $1 \mathrm{~mm}$ sodium orthovanadate, $1 \mathrm{~mm}$ glycerol phosphate) supplemented with protease inhibitor cocktail from Roche Diagnostics (Mannheim, Germany) and $10 \mathrm{~mm}$ AEBSF-Hydrochloride (AppliChem, Darmstadt, Germany). Thirty micrograms of total protein in $2 \times$ sample buffer $(100 \mathrm{~mm}$ Tris $\mathrm{pH} 6.8,200 \mathrm{~mm}$ DTT, $4 \%$ SDS, $0.05 \%$ bromphenol blue, $20 \%$ glycerol) were separated on reducing SDS-PAGE (8\%) gels and transferred to polyvinylidene fluoride membranes. Western blot analysis of FLT3 was performed with monoclonal mouse anti-human FLT3/anti-phosphoFLT3 antibodies or anti-phosphoSTAT5/anti-STAT5 antibodies followed by horseradish peroxidase-conjugated antimouse IgG antibody, and detection was done with enhanced chemiluminescence (ECL Plus Western Blotting Detection System; GE Healthcare, Chalfont, UK).

\section{Immunofluorescence Staining and Confocal \\ Immunofluorescence Microscopy}

Immunofluorescence labeling was performed according to standard procedures. In brief, COS7 cells were grown on glass coverslips coated with poly-L-lysine (Sigma Aldrich). After 12$24 \mathrm{~h}$, the plasma membrane of cells was stained on ice with WGA conjugated to Alexa Fluor 488, and thereafter cells were fixed with $4 \%$ paraformaldehyde (Merck, Darmstadt, Germany) for $10 \mathrm{~min}$ at $37^{\circ} \mathrm{C}$, quenched with $50 \mathrm{~mm}$ ammoniumchloride (Merck) for $10 \mathrm{~min}$ at room temperature, and permeabilized with $0.2 \%$ Triton X-100 (Sigma Aldrich) for $2 \mathrm{~min}$. Unspecific staining was blocked for 10 min with PBS containing 10\% FCS. Cells were incubated for $1 \mathrm{~h}$ at room temperature with primary antibodies, washed in PBS, and stained for $1 \mathrm{~h}$ in the dark with fluorophore-conjugated 
secondary antibodies. Slides were mounted in AntiFade Gold (Molecular Probes) with 1,4-diazabicyclooctane (Sigma Aldrich). Cells were examined on a confocal Olympus FV 1000 microscope (Olympus, Hamburg, Germany) with a PlanApo $60 \times / 1.4$ Oil objective or $60 \times / 1.35$ Oil objective operating in the sequential acquisition mode with 488/FITC and 543 (Cy3) or 561 excitation lasers. Fluorescent images were collected using Olympus FV 10ASW 1.6 software and processed using Adobe Photoshop 7.0.1 (Adobe Systems, San Jose, Calif., USA).

For immunofluorescence labeling to specifically stain only proteins in the plasma membrane, cells were stained for $10 \mathrm{~min}$ on ice with WGA conjugated to Alexa Fluor 488, washed with icecold PBS, and subsequently stained for $1 \mathrm{~h}$ on ice with anti-FLT3 antibody. Cells were then fixed with paraformaldehyde, quenched with $50 \mathrm{~mm}$ ammonium chloride, and stained for $1 \mathrm{~h}$ in the dark with Cy3-conjugated anti-mouse IgG antibody.

\section{Results}

The FLT3-ITD mutation is the most common gain-offunction FLT3 alteration associated with human AML. To provide insight into the mechanism of leukemic transformation induced by FLT3-ITD, we validated the expression in retrovirally transduced COS7 cells by Western blot analysis and investigated the subcellular distribution of FLT3wt and FLT3-ITD receptor proteins.

\section{Human FLT3wt and FLT3-ITD Are Expressed and}

Functional in Retrovirally Transduced COS7 Cells

In order to avoid a distinct overload of protein by transient overexpression and to achieve an expression level similar to the physiological level, we retrovirally transduced COS7 cells with either FLT3wt or FLT3-ITD. Transduction efficiency was confirmed by high-resolution denaturing fragment analysis of DNA extracted from transduced cells (fig. 1a). The FLT3-ITD signal is shifted to an increased size due to the ITD (insertion of $30 \mathrm{bp}$ ).

In order to characterize expression and activity of transduced FLT3 receptor proteins in transduced COS7 cells, we performed Western blot analysis with an antibody specifically recognizing phosphorylated FLT3 protein. FLT3-ITD is constitutively phosphorylated and therefore activated (fig. 1b, lane 4), whereas FLT3wt is phosphorylated only upon stimulation with FLT3 ligand (FL) (fig. 1b, lane 7 vs. lane 3). Total FLT3 protein was detectable in COS7 cells expressing either FLT3wt or FLT3ITD. The FLT3wt receptor protein expression level was higher than the expression level of FLT3-ITD. Several explanations are possible for this phenomenon. First, the production of retroviral supernatant was less effective for MFG-FLT3-ITD-IRES-GFP compared to MFG-FLT3wt-
IRES-GFP, because FLT3-ITD was toxic for the virusproducing cells. Resulting lower virus titers lead to less integration sites in the transduced COS7 cells and consequently to a lower expression level. In order to prove this, the number of integration sites would have to be determined by real-time PCR (Taqman).

Second, the different glycosylation pattern of FLT3ITD compared to FLT3wt receptor protein might decrease the affinity of the antibody to FLT3-ITD. Finally, the aberrant intracellular accumulation of the FLT3-ITD receptor could lead to an accelerated protein degradation or the dissolving of FLT3wt receptor protein from the plasma membrane during preparation for SDS-PAGE is more effective than the extraction of FLT3-ITD receptor protein from intracellular membranes.

Both receptor proteins showed the characteristic glycosylation pattern with FLT3wt predominantly in the mature, complex glycosylated form $(160 \mathrm{kDa})$ [Maroc et al., 1993], while FLT3-ITD receptor protein occurs mainly in the immature, high-mannose form (130 kDa) [Schmidt-Arras et al., 2005].

FLT3wt Is Located at the Plasma Membrane, whereas FLT3-ITD Accumulates in a Perinuclear Region in Retrovirally Transduced COS7 Cells

Using confocal laser scanning microscopy after performing immunocytochemistry on COS7 cells retrovirally transduced with either FLT3wt (COS7/FLT3wt) or FLT3-ITD (COS7/FLT3-ITD), we could show that in unpermeabilized cells (with the antibody having no access to the intracellular compartment) FLT3wt receptor protein was localized at the plasma membrane, whereas in FLT3-ITD-expressing cells no surface staining was detectable (fig. 2a). Consistently, when costaining FLT3 with WGA (used to label the plasma membrane) after fixation, FLT3wt was found to colocalize with WGA. In contrast, no colocalization was detectable between FLT3ITD and the plasma membrane. Instead, FLT3-ITD accumulated in a perinuclear region (fig. 2b). Also, in FLT3wt-expressing cells, additional to the surface staining, there was an intracellular accumulation, which was, however, considerably weaker than in FLT3-ITD-expressing cells.

\section{FLT3-ITD Is neither Localized in the ER nor Does It}

Accumulate in the Golgi Apparatus

It was previously reported that FLT3-ITD does not undergo complex glycosylation like the FLT3wt receptor, but persists in an underglycosylated form with highmannose structures [Schmidt-Arras et al., 2005], mean- 


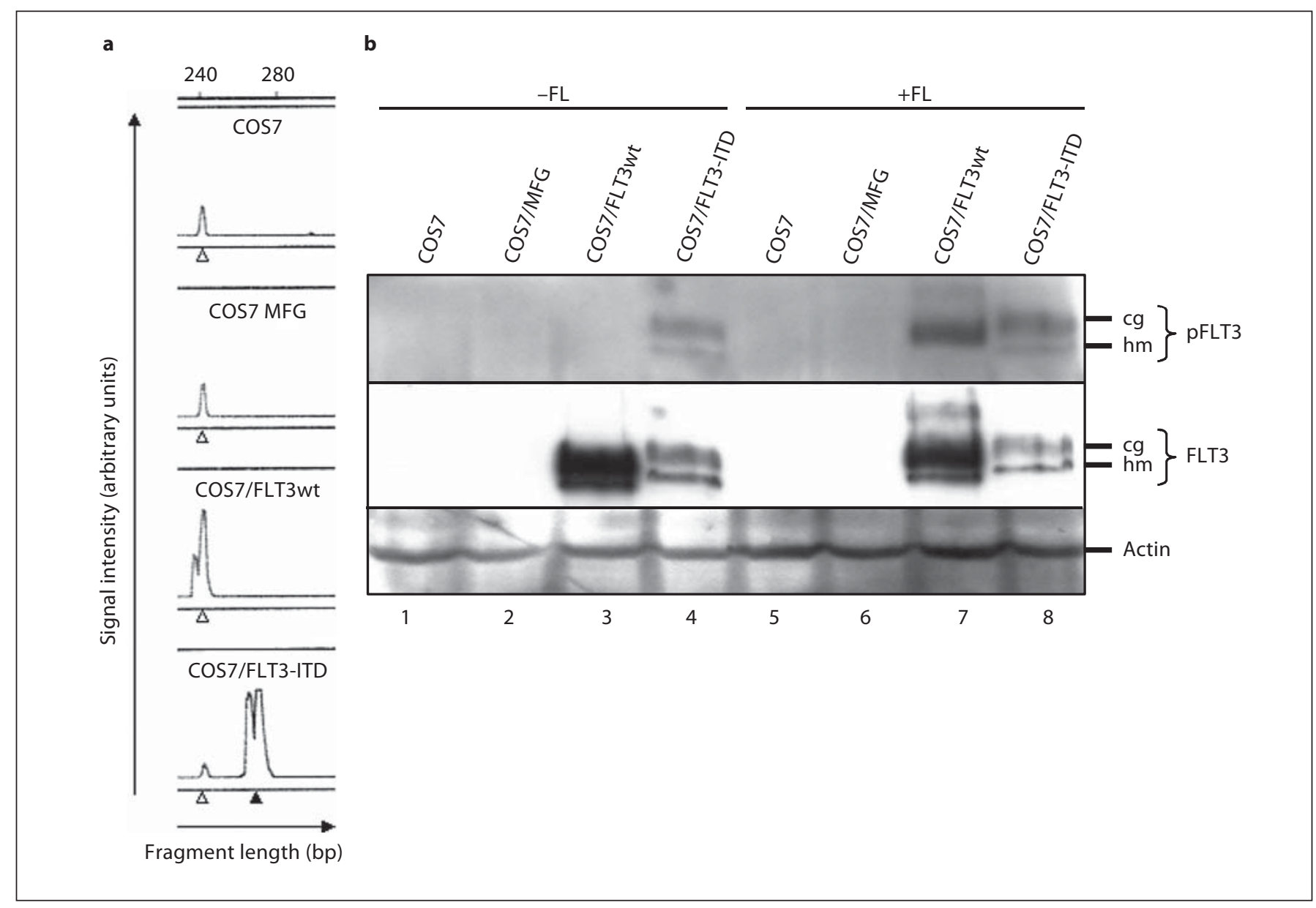

Fig. 1. Analysis of FLT3wt or FLT3-ITD expression in retrovirally transduced COS7 cells on the DNA and protein level. a Retroviral transduction of FLT3wt and FLT3-ITD into COS7 cells was confirmed by high-resolution denaturing fragment analysis. Electropherograms show the size of the PCR product in base pairs (x-axis) and fluorescence units (y-axis) which are proportional to the amount of the PCR product. $\triangle$ = FLT3wt; $\boldsymbol{\Delta}=$ FLT3-ITD. b Functionality of stably expressed receptor proteins was confirmed by Western blot analysis. Cells were not starved or serum

Fig. 2. Localization of FLT3wt and FLT3-ITD. COS7 cells retrovirally transduced with either FLT3wt (COS7/FLT3wt) or FLT3ITD (COS7/FLT3-ITD) were processed for confocal imaging with indicated reagent and antibodies. a Immunostaining prior to fixation and without permeabilization with an anti-FLT3 antibody against an extracellular epitope of FLT3 to visualize only those FLT3 receptor proteins which are present in the plasma membrane. FLT3 is detectable at the plasma membrane only in COS7/ FLT3wt cells. b Immunostaining after fixation and permeabilization to allow antibodies access to the intracellular part of the cells. While FLT3wt colocalizes with WGA, FLT3-ITD does not colocalize with WGA, but accumulates in a perinuclear region. c COS7 cells transiently overexpressing FLT3wt, additional to the starved for $16 \mathrm{~h}$ and stimulated with $100 \mathrm{ng}$ FLT3 ligand/ml for $5 \mathrm{~min}$. Stably expressed FLT3-ITD receptor is constitutively phosphorylated, while FLT3wt receptor is phosphorylated only upon FLT3 ligand stimulation. After immunostaining with phosphospecific antibodies, blots were stripped and reprobed with respective antibodies for the total protein. Actin was used as a loading control to confirm equal protein loading. The presented Western blot is a representative example of 3 independent experiments. $\mathrm{cg}=$ Complex glycosilated; $\mathrm{hm}=$ high-mannose; FL = FLT3 ligand .

plasma membrane staining, show a perinuclear accumulation similar to FLT3-ITD (upper panel). Transiently overexpressed FLT3wt protein accumulates in the ER (lower panel, white arrowhead). d Cells were costained for FLT3 and endogenous calnexin. While a minor part of FLT3-ITD colocalizes with calnexin, the main clusters of FLT3-ITD are devoid of calnexin (white arrowheads). e Cells were costained for FLT3 and endogenous giantin. Neither FLT3wt nor FLT3-ITD colocalize with giantin. Images are representative examples of 3 independent experiments. In each of the experiments, 3 images with cells showing the typical staining pattern were scanned as representative examples. Scale bars correspond to $10 \mu \mathrm{m}$.

(For figures see next pages.) 

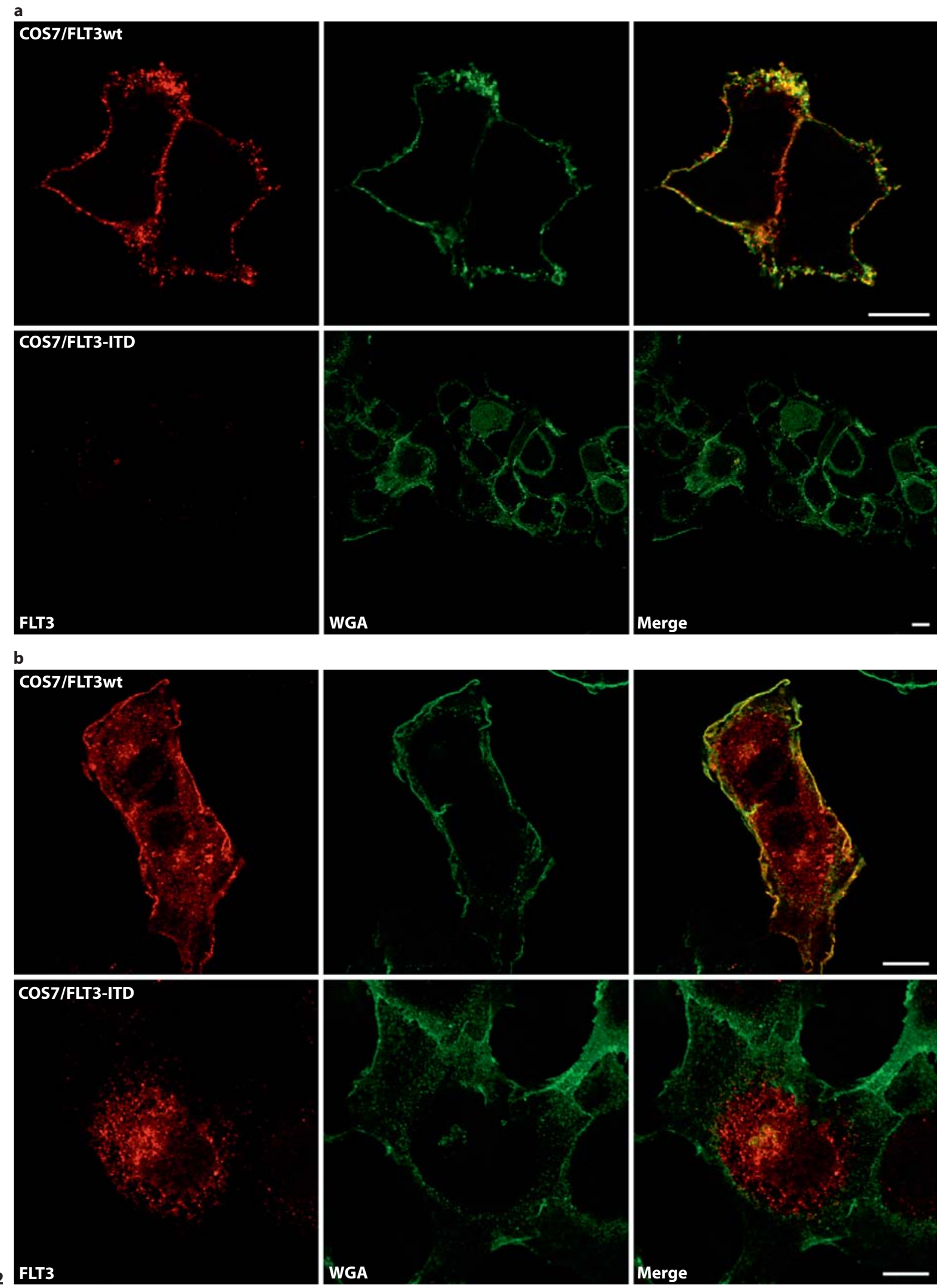

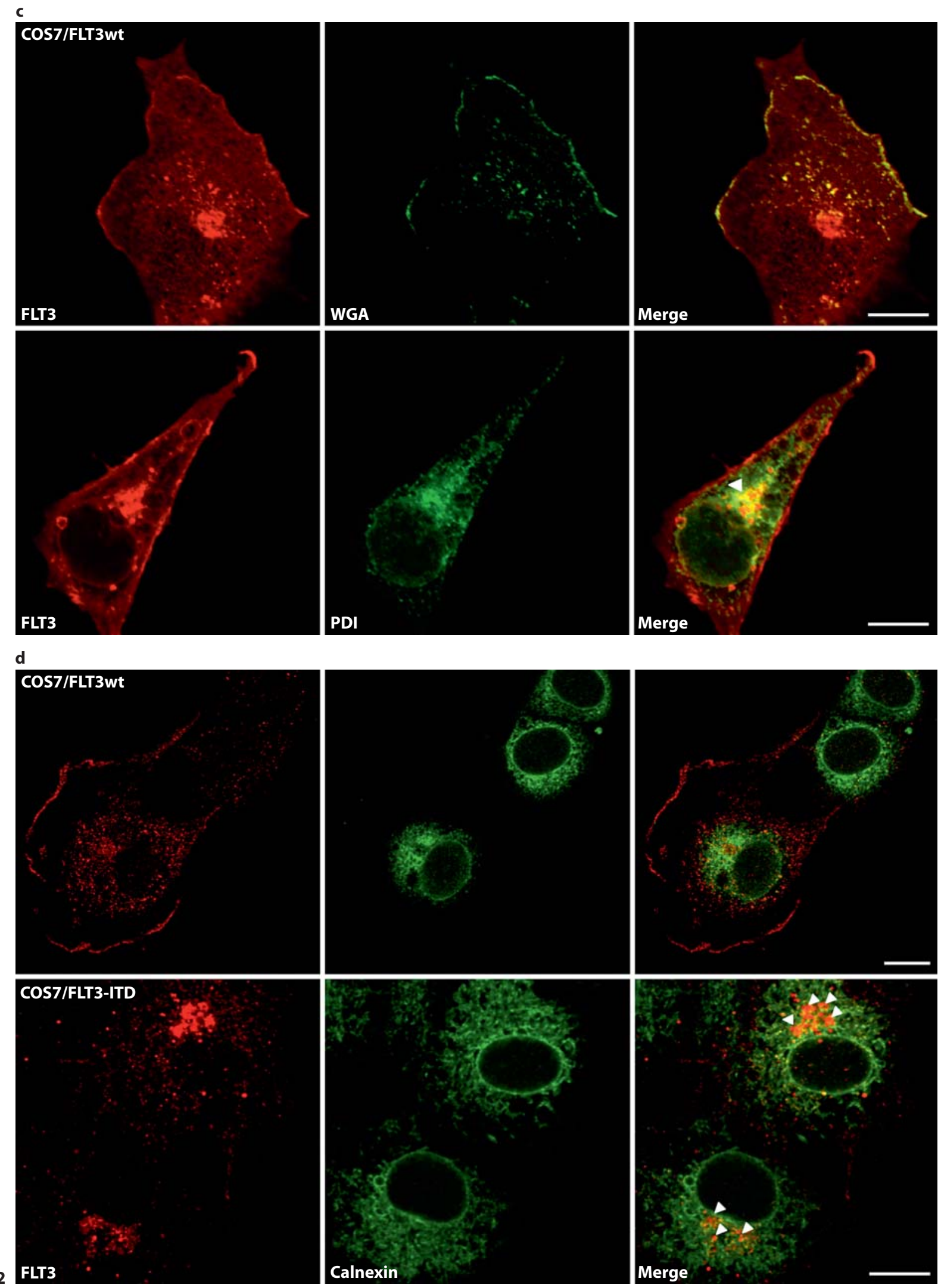

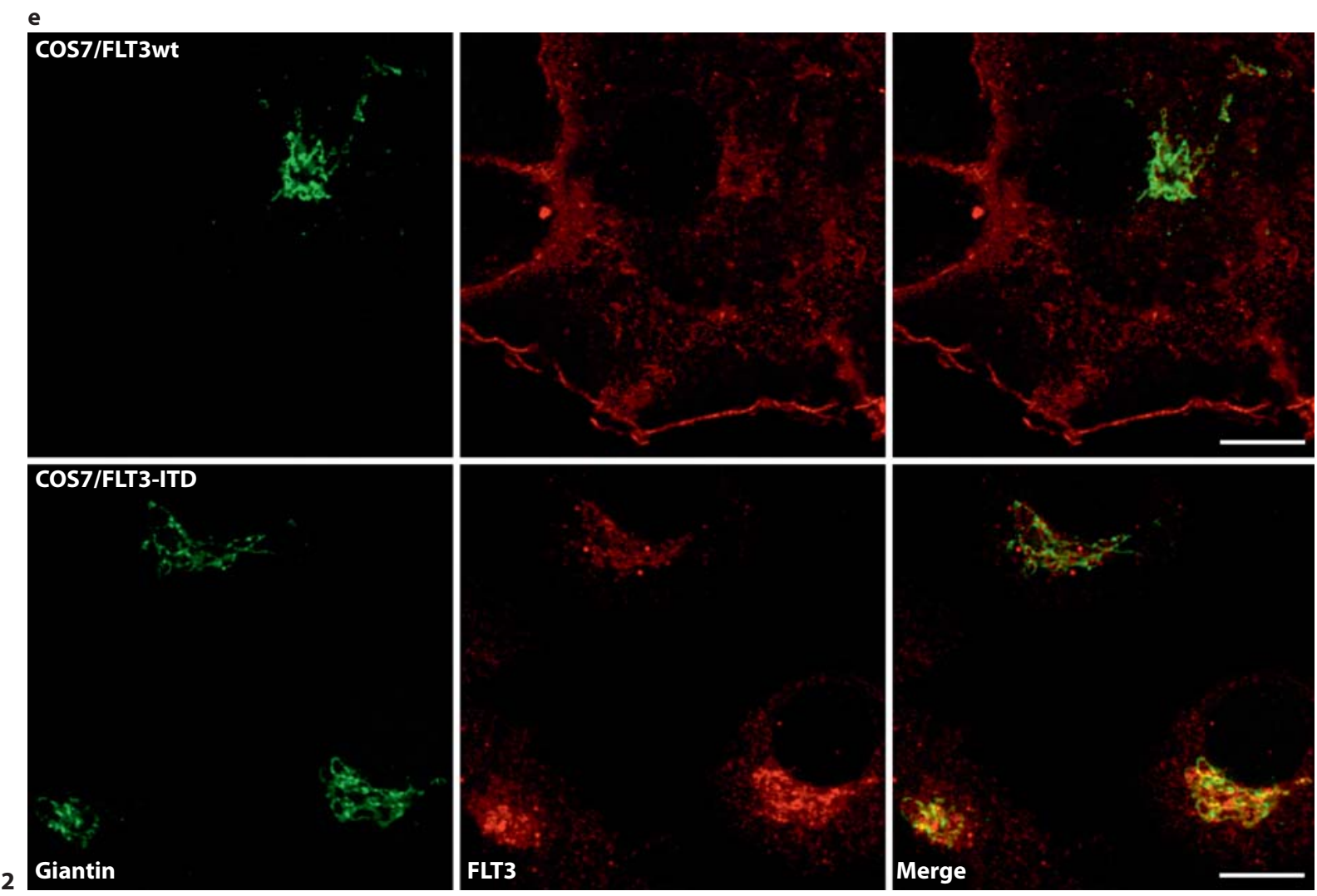

ing that ER-specific glycosylation is present in FLT3-ITD molecules, whereas Golgi-specific glycosylation is only present in FLT3wt molecules. Additionally, this study showed that transiently overexpressed FLT3-ITD colocalized both with an overexpressed ER-targeting sequence derived from calreticulin, including a KDEL, fused to enhanced cyan fluorescent protein (ECFP) and with an overexpressed Golgi compartment-targeting sequence derived from human $\beta$-1,4-galactosyltransferase fused to ECFP [Schmidt-Arras et al., 2005]. Indeed, when transiently overexpressed, even FLT3wt accumulates intracellularly in addition to its predominant surface localization (fig. 2c, upper panel) and colocalizes with endogenous PDI (ER marker) in COS7 cells (fig. 2c, lower panel). This result shows that transient overexpression might lead to an overload of protein which exceeds the capacity of the ER sorting machinery. In contrast, costaining of stably expressed FLT3 with endogenous calnexin (ER marker) revealed that the main part of the FLT3-ITDcontaining intracellular compartment does not represent the ER (fig. 2d).

Since FLT3-ITD did not colocalize with calnexin, we searched for another cell organelle as the place of FLT3-
ITD accumulation. In the secretory pathway, the organelle subsequent to the ER is the Golgi apparatus. There is one study which shows a constitutively active KIT mutant to accumulate in the Golgi apparatus [Xiang et al., 2007]. Therefore, we costained FLT3 with an antibody against endogenous giantin, a marker for the Golgi apparatus. Unexpectedly, we could not observe any colocalization of FLT3-ITD with giantin (fig. 2e) in 3 independent experiments. These results suggest that FLT3-ITD is neither localized in the ER nor in the Golgi apparatus, but accumulates in a so far unidentified, additional compartment.

\section{Discussion}

Activating mutations in RTKs occur commonly in cancer. The mechanisms by which mutant RTKs contribute to neoplasia are not fully understood. Particular mutations might not only induce intracellular tyrosine phosphorylation and kinase activity, but also affect subcellular localization of the receptor, which in turn might impair the activation of downstream substrates by providing access to additional/different substrates. 
Up to now, there are only a few reports about an aberrant intracellular localization of mutated RTKs which point to the importance of this phenomenon as a mechanism for pathological signaling [Miettinen and Lasota, 2001; Clarke and Dirks, 2003; Xiang et al., 2007]. For instance, a human glioblastoma-derived constitutively active PDGFR $\alpha$ mutant is localized predominantly in the cytoplasm, while in contrast, wild-type PDGFR $\alpha$ is mainly expressed at the cell surface, although additionally detectable in the cytoplasm [Clarke and Dirks, 2003]. The authors suggest that abundance of mutant PDGFR $\alpha$ in the cytoplasm may enable the mutant receptors to activate distinct downstream signaling intermediates. Another example is KIT localization in human gastrointestinal stromal tumors, which is described as pancytoplasmic or as perinuclear dots (Golgi zone pattern) [Miettinen and Lasota, 2001]. Furthermore, in a murine model of the constitutively active human $\mathrm{KIT}^{\mathrm{D} 816 \mathrm{~V}}$ mutant-mediated leukemia, the mutant receptor protein was retained in the ER, whereas in human A375 cells it was localized in the Golgi apparatus and to a lesser extent to the plasma membrane [Xiang et al., 2007]. In this study, the authors describe a link between cellular transformation and intracellular localization, that is, ER-localized human $\mathrm{KIT}^{\mathrm{D} 816 \mathrm{~V}}$ failed to induce myeloproliferative diseases in mice, while Golgi-localized mouse-human hybrid $\mathrm{KIT}^{\mathrm{D} 816 \mathrm{~V}}$ or cytosol-localized, ectodomain-deleted $\mathrm{KIT}^{\mathrm{D} 816 \mathrm{~V}}$ were able to cause fatal myeloproliferative diseases in mice. Taken together, these examples provide evidence for the striking importance of subcellular localization of RTKs for the functional outcome.

So far, there is only one study investigating the phosphorylation of FLT3-ITD and other RTKs results in aberrant intracellular receptor trafficking with entrapment of phosphorylated RTKs in the ER [Schmidt-Arras et al., 2005]. In our study, we confirm aberrant intracellular accumulation of FLT3-ITD. In addition, we compared FLT3-ITD staining to FLT3wt staining, the latter being predominantly localized at the cell surface. Nevertheless, we could show for the first time by immunofluorescence staining and confocal microscopy that FLT3wt was also detectable in a perinuclear region, although to a weaker extent than FLT3-ITD. This might be due to a slight overexpression of FLT3wt, which still occurs with retroviral transduction, and the sorting machinery of the secretory pathway might be overloaded with the protein, but it is also likely to reflect the physiological localization of FLT3wt. This is supported by preliminary data from primary human CD34+ cells, where FLT3wt receptor protein is detectable intracellularly in addition to its surface localization [Koch et al., unpubl. observations]. In contrast to Schmidt-Arras et al. [2005], we could demonstrate that the intracellular accumulation of FLT3-ITD does neither reflect colocalization with the ER marker calnexin nor with the Golgi marker giantin. Reasons for these observed differences might be that in the study of Schmidt-Arras et al. [2005], both FLT3-ITD construct and organelle markers were transiently transfected into COS7 cells, leading to a distinct protein overexpression of both FLT3-ITD and organelle markers. Furthermore, to label the organelles, fusion constructs of an organelleresident protein with ECFP were used. An expression construct containing ECFP fused to an ER-targeting sequence derived from calreticulin, including a KDEL retrieval sequence, was used as an ER localization marker. ECFP fused to a Golgi compartment-targeting sequence derived from human $\beta$-1,4-galactosyltransferase was used as a Golgi compartment localization marker. Both overexpression and tagging of proteins of interest are known to potentially alter the intracellular localization or the function of the target protein [Miyashita, 2004] and both might be the cause of the different observations described here.

In contrast, in our study, we retrovirally transduced both FLT3wt or FLT3-ITD into COS7 cells resulting in expression levels of FLT3 receptor protein resembling the physiological levels. As confirmed by Western blot analysis, both FLT3wt and FLT3-ITD receptor proteins are functional in transduced COS7 cells (fig. 1). Additionally, and in contrast to Schmidt-Arras et al. [2005], we used antibodies against endogenous marker proteins for the ER (calnexin) and Golgi apparatus (giantin). In order to prove that localization is altered by overexpression per se, we transiently transfected COS7 cells with FLT3wt. Using this mode of transgene expression, we found overexpressed FLT3wt receptor protein to be localized in the ER (fig. 2c).

Biochemically, we observed the previously described 2 distinct pools of FLT3-ITD receptor: immature highmannose receptor protein and a fainter signal for the mature, complex glycosylated receptor [Schmidt-Arras et al., 2005], which was probably too weak to be detected at the cell surface by immunofluorescence microscopy. This is consistent with the model that constitutively activated FLT3-ITD receptor proteins, which fail to achieve a mature glycosylation pattern, do not traffic normally through the secretory pathway. This failure seems to be substantial but not complete.

In conclusion, we propose a model in which the constitutively active immature FLT3-ITD receptor is aber- 
rantly accumulating intracellularly in an unidentified compartment of the secretory pathway. From its intracellular location, it might be able to recruit additional or different signaling pathways compared to the predominantly surface-localized FLT3wt enabling transformation of the cell.

It remains to be clarified in which intracellular compartment FLT3-ITD is accumulating. We could show that FLT3-ITD accumulates intracellularly, but the place of accumulation is neither the ER nor the Golgi apparatus. Additionally, FLT3 is a membrane-spanning protein which has to pass the secretory pathway, and the glycosylation pattern provides evidence that FLT3-ITD most probably resides somewhere before or in the Golgi apparatus. Thus, possible further organelles or subcompartments of organelles to test for FLT3-ITD presence might be the ER exit sites, the ER-Golgi intermediate compartment or the trans-Golgi network [Schülein, 2004; Kitzberger et al., 2005; Watson et al., 2006].
Furthermore, the functional consequences of aberrant localization/trafficking have to be confirmed. Does intracellular retention of mutant RTKs change the quality of signal transduction by providing access to substrates which are not available for RTKs at the cell membrane and/or does increased intracellular retention of mutant RTKs alter the level of signal transduction rather than its quality? These future observations may be relevant to the treatment of human cancers driven by mutant RTKs. Understanding the molecular mechanism by which mutant FLT3-ITD activates disease-causing downstream signaling pathway(s) will potentially allow us to identify and evaluate novel therapeutic strategies.

\section{Acknowledgments}

This work was supported in part by funding from the SFB655 projects A16 (C.T.) and A17 (G.E.). We thank Marika Karger for excellent technical assistance.

\section{References}

Antonescu, C.R., G. Sommer, L. Sarran, S.J. Tschernyavsky, E. Riedel, J.M. Woodruff, M. Robson, R. Maki, M.F. Brennan, M. Ladanyi, R.P. Dematteo, P. Besmer (2003) Association of KIT exon 9 mutations with nongastric primary site and aggressive behavior: KIT mutation analysis and clinical correlates of 120 gastrointestinal stromal tumors. Clin Cancer Res 9: 3329-3337.

Blume-Jensen, P., T. Hunter (2001) Oncogenic kinase signalling. Nature 411: 355-365.

Choudhary, C., J. Schwable, C. Brandts, L. Tickenbrock, B. Sargin, T. Kindler, T. Fischer, W.E. Berdel, C. Muller-Tidow, H. Serve (2005) AML-associated Flt3 kinase domain mutations show signal transduction differences compared with Flt3 ITD mutations. Blood 106: 265-273.

Clarke, I.D., P.B. Dirks (2003) A human brain tumor-derived PDGFR- $\alpha$ deletion mutant is transforming. Oncogene 22: 722-733.

Cosset, F.L., Y. Takeuchi., J.L. Battini, R.A. Weiss, M.K. Collins (1995) High-titer packaging cells producing recombinant retroviruses resistant to human serum. J Virol 69: 7430-7436.

Cui, J., B.I. Yang, X.J. Bi, Z.R. Fan (2001) Methylation status of $\mathrm{c}$-fms oncogene in HCC and its relationship with clinical pathology. World J Gastroenterol 7: 136-139.

Grassot, J., G. Mouchiroud, G. Perriere (2003) RTKdb: database of Receptor Tyrosine Kinase. Nucleic Acids Res 31: 353-358.
Griffith, J., J. Black, C. Faerman, L. Swenson, M. Wynn, F. Lu, J. Lippke, K. Saxena (2004) The structural basis for autoinhibition of FLT3 by the juxtamembrane domain. Mol Cell 13: 169-178.

Grundler, R., C. Miething, C. Thiede, C. Peschel, J. Duyster (2005) FLT3-ITD and tyrosine kinase domain mutants induce 2 distinct phenotypes in a murine bone marrow transplantation model. Blood 105: 4792-4799.

Heinrich, M.C., C.L. Corless, G.D. Demetri, C.D. Blanke, M. von Mehren, H. Joensuu, L.S. Mcgreevey, C.J. Chen, A.D. van den Abbeele, B.J. Druker, B. Kiese, B. Eisenberg, P.J. Roberts, S. Singer, C.D. Fletcher, S. Silberman, S. Dimitrijevic, J.A. Fletcher (2003) Kinase mutations and imatinib response in patients with metastatic gastrointestinal stromal tumor. J Clin Oncol 21: 4342-4349.

Heldin, C.H. (1995) Dimerization of cell surface receptors in signal transduction. Cell 80: 213-223.

Huse, M., J. Kuriyan (2002) The conformational plasticity of protein kinases. Cell 109: 275282.

Kacinski, B.M., K.A. Scata, D. Carter, L.D. Yee, E. Sapi, B.L. King, S.K. Chambers, M.A. Jones, M.H. Pirro, E.R. Stanley, et al. (1991) FMS (CSF-1 receptor) and CSF-1 transcripts and protein are expressed by human breast carcinomas in vivo and in vitro. Oncogene 6: 941-952.
Kirma, N., L.S. Hammes, Y.G. Liu, H.B. Nair, P. T. Valente,S. Kumar, L.C. Flowers, R.R. Tekmal (2007) Elevated expression of the oncogene $\mathrm{c}-\mathrm{fms}$ and its ligand, the macrophage colony-stimulating factor-1, in cervical cancer and the role of transforming growth factor-betal in inducing c-fms expression. Cancer Res 67: 1918-1926.

Kitzberger, R., C. Madl, P. Ferenci (2005) Wilson disease. Metab Brain Dis 20: 295-302.

Kiyoi, H., R. Ohno, R. Ueda, H. Saito, T. Naoe (2002) Mechanism of constitutive activation of FLT3 with internal tandem duplication in the juxtamembrane domain. Oncogene 21: 2555-2563.

Kumabe, T., Y. Sohma, T. Kayama, T. Yoshimoto, T. Yamamoto (1992) Overexpression and amplification of $\alpha$-PDGF receptor gene lacking exons coding for a portion of the extracellular region in a malignant glioma. Tohoku J Exp Med 168: 265-269.

Longley, B.J., L. Tyrrell, S.Z. Lu, Y.S. Ma, K. Langley, T.G. Ding, T. Duffy, P. Jacobs, L.H. Tang, I. Modlin (1996) Somatic c-KIT activating mutation in urticaria pigmentosa and aggressive mastocytosis: establishment of clonality in a human mast cell neoplasm. Nat Genet 12: 312-314.

Maroc, N., R. Rottapel, O. Rosnet, S. Marchetto, C. Lavezzi, P. Mannoni, D. Birnbaum, P. Dubreuil (1993) Biochemical characterization and analysis of the transforming potential of the FLT3/FLK2 receptor tyrosine kinase. Oncogene 8: 909-918. 
Miettinen, M., J. Lasota (2001) Gastrointestinal stromal tumors - definition, clinical, histological, immunohistochemical, and molecular genetic features and differential diagnosis. Virchows Arch 438: 1-12.

Miyashita, T. (2004) Confocal microscopy for intracellular co-localization of proteins. Methods Mol Biol 261: 399-410.

Mol, C.D., D.R. Dougan, T.R. Schneider, R.J. Skene, M.L. Kraus, D.D. Scheibe, G.P. Snell, H. Zou, B.C. Sang, K.P. Wilson (2004) Structural basis for the autoinhibition and STI571 inhibition of c-Kit tyrosine kinase. J Biol Chem 279: 31655-31663.

Nagata, H., A.S. Worobec, C.K. Oh, B.A. Chowdhury, S. Tannenbaum, Y. Suzuki, D.D. Metcalfe (1995) Identification of a point mutation in the catalytic domain of the protooncogene c-kit in peripheral blood mononuclear cells of patients who have mastocytosis with an associated hematologic disorder. Proc Natl Acad Sci USA 92: 10560 10564.

Riviere, I., K. Brose, R.C. Mulligan (1995) Effects of retroviral vector design on expression of human adenosine deaminase in murine bone marrow transplant recipients engrafted with genetically modified cells. Proc Nat Acad Sci USA 92: 6733-6737.

Robinson, D.R., Y.M. Wu, S.F. Lin (2000) The protein tyrosine kinase family of the human genome. Oncogene 19: 5548-5557.

Rosnet, O., D. Birnbaum (1993) Hematopoietic receptors of class III receptor-type tyrosine kinases. Crit Rev Oncog 4: 595-613.
Rubin, B.P., S. Singer, C. Tsao, A. Duensing, M. L. Lux, R. Ruiz, M.K. Hibbard, C.J. Chen, S. Xiao, D.A. Tuveson, G.D. Demetri, C.D. Fletcher, J.A. Fletcher (2001) KIT activation is a ubiquitous feature of gastrointestinal stromal tumors. Cancer Res 61: 8118-8121.

Schmidt-Arras, D.E., A. Bohmer, B. Markova, C. Choudhary, H. Serve, F.D. Bohmer (2005) Tyrosine phosphorylation regulates maturation of receptor tyrosine kinases. Mol Cell Biol 25: 3690-3703.

Schülein, R. (2004) The early stages of the intracellular transport of membrane proteins: clinical and pharmacological implications. Rev Physiol Biochem Pharmacol 151: 45-91.

Tang, R.P., B. Kacinski, P. Validire, F. Beuvon, X. Sastre, P. Benoit, A. Dela Rochefordiere, V. Mosseri, P. Pouillart, S. Scholl (1990) Oncogene amplification correlates with dense lymphocyte infiltration in human breast cancers: a role for hematopoietic growth factor release by tumor cells? J Cell Biochem 44 : 189-198.

Tobal, K., A. Pagliuca, B. Bhatt, N. Bailey, D.M. Layton, G.J. Mufti (1990) Mutation of the human FMS gene (M-CSF receptor) in myelodysplastic syndromes and acute myeloid leukemia. Leukemia 4: 486-489.

Turner, A.M., N.L. Lin, S. Issarachai, S.D. Lyman, V.C. Broudy (1996) FLT3 receptor expression on the surface of normal and malignant human hematopoietic cells. Blood 88 : $3383-3390$
Van der Geer, P., T. Hunter, R.A. Lindberg (1994) Receptor protein-tyrosine kinases and their signal transduction pathways. Annu Rev Cell Biol 10: 251-337.

Walter, M., I.S. Lucet, O. Patel, S.E. Broughton, R. Bamert, N.K. Williams, E. Fantino, A.F. Wilks, J. Rossjohn (2007) The 2.7 A crystal structure of the autoinhibited human c-Fms kinase domain. J Mol Biol 367: 839-847.

Watson, P., A.K. Townley, P. Koka, K.J. Palmer, D.J. Stephens (2006) Sec16 defines endoplasmic reticulum exit sites and is required for secretory cargo export in mammalian cells. Traffic 7: 1678-1687.

Xiang, Z.,F. Kreisel, J. Cain, A. Colson, M.H. Tomasson (2007) Neoplasia driven by mutant c-KIT is mediated by intracellular, not plasma membrane, receptor signalling. Mol Cell Biol 27: 267-282.

Yamamoto, Y., H. Kiyoi, Y. Nakano, R. Suzuki, Y. Kodera, S. Miyawaki, N. Asou, K. Kuriyama, F. Yagasaki, C. Shimazaki, H. Akiyama, K. Saito, M. Nishimura, T. Motoji, K. Shinagawa, A. Takeshita, H. Saito, R. Ueda, R. Ohno, T. Naoe (2001) Activating mutation of D835 within the activation loop of FLT3 in human hematologic malignancies. Blood 97: 2434-2439.

Yang, D.H., W. Huang, J. CUI, K.C. Shu, S.H. Tang, W.J. Zhang, J.H. Liang (2004) The relationship between point mutation and abnormal expression of $\mathrm{c}$-fms oncogene in hepatocellular carcinoma. Hepatobiliary Pancreat Dis Int 3: 86-89. 